\title{
The excitation of 5-min oscillations in the solar corona
}

\author{
T. V. Zaqarashvili ${ }^{1,4}$, K. Murawski ${ }^{2}$, M. L. Khodachenko ${ }^{1}$, and D. Lee ${ }^{3}$ \\ 1 Space Research Institute, Austrian Academy of Sciences, Schmiedlstrasse 6, 8042 Graz, Austria \\ e-mail: [teimuraz.zaqarashvili;maxim.khodachenko]@oeaw.ac.at \\ 2 Group of Astrophysics, Institute of Physics, UMCS, ul. Radziszewskiego 10, $20-031$ Lublin, Poland \\ e-mail: kmur@kft.umcs.lublin.pl \\ 3 ASC/Flash Center, The University of Chicago, 5640 S. Ellis Ave, Chicago, IL 60637, USA \\ e-mail: dongwook@flash.uchicago.edu \\ 4 Abastumani Astrophysical Observatory at Ilia State University, Kazbegi ave. 2a, Tbilisi, Georgia
}

Received 13 July 2010 / Accepted 28 January 2011

\begin{abstract}
Aims. We aim to study excitation of the observed $\sim 5$-min oscillations in the solar corona by localized pulses that are launched in the photosphere.

Methods. We solve the full set of nonlinear one-dimensional Euler equations numerically for the velocity pulse propagating in the solar atmosphere that is determined by the realistic temperature profile.

Results. Numerical simulations show that an initial velocity pulse quickly steepens into a leading shock, while the nonlinear wake in the chromosphere leads to the formation of consecutive pulses. The time interval between the arrivals of two neighboring pulses to a detection point in the corona is approximately $5 \mathrm{~min}$. Therefore, the consecutive pulses may result in the $\sim 5$-min oscillations that are observed in the solar corona.

Conclusions. The $\sim 5$-min oscillations observed in the solar corona can be explained in terms of consecutive shocks that result from impulsive triggers launched within the solar photosphere by granulation and/or reconnection.
\end{abstract}

Key words. Sun: atmosphere - Sun: oscillations

\section{Introduction}

Propagating acoustic waves are frequently seen in the solar corona as periodic variations in the spectral line intensity (De Moortel et al. 2000, 2002; Marsh et al. 2003; Lin et al. 2005, 2006; Srivastava et al. 2008; Wang et al. 2009). As these waves are often observed within the frequency range corresponding to acoustic waves in the solar photosphere/chromosphere, this logically implies that the photospheric acoustic oscillations penetrate into the corona. However, the photospheric 5-min oscillations are evanescent in the gravitationally stratified solar atmosphere as their frequency is lower than the cut-off frequency (Lamb 1908; Roberts 2004; Musielak et al. 2006). Bel \& Leroy (1977) suggested that the cut-off frequency of the magnetic fieldfree atmosphere is lower for waves propagating obliquely to the vertical direction. De Pontieu et al. (2005) proposed that p-modes might be channeled into the solar corona along inclined magnetic field lines as a result of the decrease in the acoustic cut-off frequency. McIntosh \& Jefferies (2006) found the observational justification the cut-off frequency had been modified by the inclined magnetic field. As the magnetic field of active region loops is predominantly vertical in the photosphere/chromosphere, it is unclear how p-modes may penetrate into the coronal regions.

There are two different types of drivers in the highly dynamic solar photosphere: oscillatory (e.g. p-modes) and impulsive (e.g. granulation and/or explosive events due to magnetic reconnection). Both types of drivers may be responsible for the observed dynamical phenomena in upper atmospheric regions.

As a result of the rapid decrease in the mass density with height, finite-amplitude high-frequency photospheric oscillations can quickly grow in their amplitudes and steepen into shocks, which by energy dissipation can lead to the chromospheric heating (Narain \& Ulmschneider 1990, 1996; Carlsson \& Stein 1997; Ruderman 2006). Lower-frequency waves, those with a $\sim 5$ min period, are not good candidates for chromospheric heating (Narain \& Ulmschneider 1990).

It was found by Hollweg (1982) that a localized pulse launched initially sets up a nonlinear wake leading to a trail of consecutive shocks. These shocks were called rebound shocks by Hollweg (1982).

The time interval between consecutive shocks is close to the period of the nonlinear wake. In the linear case, the wake oscillates with the acoustic cut-off frequency of the stratified atmosphere. Nonlinearity modifies the wave period of the wake, with many features of spicules exhibiting the periodicity of about 5-min (Murawski \& Zaqarashvili 2010). These quasi-periodic shocks may then lead to the oscillatory dynamics of coronal plasma, which is observed as intensity oscillations in coronal spectral lines.

The aim of this paper is to study the effect of rebound shocks, which are formed by an impulsive perturbation, on the observed $\sim 5$-min oscillations in the solar corona. We consider the simplest hydrodynamic case, which can be developed into a more realistic magnetohydrodynamic model in future studies.

This paper is organized as follows. The basic equations and the atmospheric model are described in Sect. 2. The numerical model and results of numerical simulations of impulsive photospheric driver are discussed in Sect. 3. This paper is concluded by a summary of the main results in Sect. 4 . 


\section{Basic model}

\subsection{Hydrodynamic equations}

Our model system is taken to be composed of a gravitationallystratified solar atmosphere that is described by one-dimensional (1D) Euler equations

$\frac{\partial \varrho}{\partial t}+\frac{\partial(\varrho V)}{\partial y}=0$

$\varrho \frac{\partial V}{\partial t}+\varrho V \frac{\partial V}{\partial y}=-\frac{\partial p}{\partial y}-\varrho g$

$\frac{\partial p}{\partial t}+\frac{\partial(p V)}{\partial y}=(1-\gamma) p \frac{\partial V}{\partial y}$

where $\varrho$ denotes the mass density, $V$ is a vertical component of the flow velocity, $p=k_{\mathrm{B}} \varrho T / m$ is the gas pressure, $\gamma=5 / 3$ is the adiabatic index, $g=272 \mathrm{~m} \mathrm{~s}^{-2}$ is the gravitational acceleration, $T$ is the temperature, $m$ is the mean particle mass, and $k_{\mathrm{B}}$ is Boltzmann's constant.

\subsection{The equilibrium}

We assume that in equilibrium the solar atmosphere is settled in a static $(V=0)$ environment in which the pressure gradient force is balanced by the gravity, that is

$-\frac{\partial p_{0}}{\partial y}-\varrho_{0} g=0$

With the use of the equation of state, we obtain the equilibrium gas pressure and mass density as

$p_{0}(y)=p_{00} \exp \left(-\int_{y_{\mathrm{r}}}^{y} \frac{\mathrm{d} y^{\prime}}{\Lambda\left(y^{\prime}\right)}\right), \quad \varrho_{0}(y)=\frac{p_{0}(y)}{g \Lambda(y)}$,

where $\Lambda(y)=k_{\mathrm{B}} T(y) /(m g)$ is the pressure scale-height and $p_{00}$ is the gas pressure at the reference level, chosen here at $y_{\mathrm{r}}=$ $10 \mathrm{Mm}$.

We adopt a realistic temperature profile $T(y)$ for the solar atmosphere (Vernazza et al. 1981). In this profile, $T$ attains a value of about $5700 \mathrm{~K}$ at the top of the photosphere, which corresponds to $y=0.5 \mathrm{Mm}$. At higher altitudes, $T(y)$ falls off until it reaches its minimum of $4350 \mathrm{~K}$ at $y \simeq 0.95 \mathrm{Mm}$. At yet higher altitudes, $T(y)$ grows gradually with height to the transition region, which is located at $y \simeq 2.7 \mathrm{Mm}$. Here $T(y)$ undergoes rapid growth to the coronal value of $1.5 \mathrm{MK}$ at $y=10 \mathrm{Mm}$. After specifying $T(y)$, we can obtain mass density and gas pressure profiles with the use of Eq. (5).

We solve Eqs. (1)-(3) numerically for an impulsive perturbation launched initially within the solar photosphere. The numerical simulations for a harmonic driver have been reported elsewhere (e.g. Erdélyi et al. 2007; Fedun et al. 2009, and references therein). Therefore, it is justifiable to limit our study to the case of impulsively generated waves.

\subsection{Linear approximation}

Neglecting all nonlinear terms in Eqs. (1)-(3) leads to the classical Klein-Gordon equation (Rae \& Roberts 1982; Roberts 2004)

$\frac{\partial^{2} Q}{\partial t^{2}}-c_{\mathrm{s}}^{2} \frac{\partial^{2} Q}{\partial y^{2}}+\Omega_{\mathrm{c}}^{2} Q=0$ where $Q(t, y)=V(t, y) \sqrt{\varrho_{0}(0) c_{\mathrm{s}}^{2}(0) / \varrho_{0}(y) c_{\mathrm{s}}^{2}(y)}$ and

$\Omega_{\mathrm{c}}^{2}(y)=\frac{c_{\mathrm{s}}^{2}}{4 \Lambda}\left(1+2 \frac{\partial \Lambda}{\partial y}\right)$.

For an isothermal atmosphere, the Klein-Gordon equation implies a natural frequency of the stratified medium, $\Omega_{\mathrm{c}}=c_{\mathrm{s}} / 2 \Lambda$, which is called the cut-off frequency for acoustic waves. A physical interpretation of the cut-off frequency is that waves of lower (higher) frequencies than $\Omega_{\mathrm{c}}$ are evanescent (propagating). According to the theory developed for the linear Klein-Gordon equation, the initially launched pulse results in a leading pulse that propagates with the sound speed. This pulse is followed by the wake, which oscillates with $\Omega_{\mathrm{c}}$ and gradually decays as time progresses (Lamb 1908; Rae \& Roberts 1982). However, the situation is different when one considers nonlinear terms in Eqs. (1)-(3). A large-amplitude initial pulse may then lead to consecutive shocks due to nonlinearity (Hollweg 1982). In the nonlinear regime, the wake may oscillate with the period, which differs from the linear cut-off period. The interval between consecutive shocks may then depend on the amplitude of the initial pulse (Murawski \& Zaqarashvili 2010). We solve the fully nonlinear equations for a realistic atmospheric model numerically and the results are presented in the next section.

\section{Numerical simulations for impulsively generated waves}

Equations (1)-(3) are solved by using the code FLASH (Dubey et al. 2009). We define the simulation region to be $-0.5 \mathrm{Mm} \leq$ $y \leq 29.5 \mathrm{Mm}$ and at the numerical boundaries we fix in time all plasma quantities to their equilibrium values. In our studies, we use an adaptive mesh refinement (AMR) grid with a minimum (maximum) level of refinement blocks, which is set to 3 (9). In the simulations, we excite waves by launching at $t=0 \mathrm{~s}$ the initial velocity pulse of a Gaussian profile

$V(y, t=0)=A_{\mathrm{v}} \exp \left[-\frac{\left(y-y_{0}\right)^{2}}{w^{2}}\right]$,

where $A_{\mathrm{v}}$ is the amplitude of the initial pulse, $y_{0}$ is its initial position, and $w$ is its width. We set and hold fixed $y_{0}=0.5 \mathrm{Mm}$ and $w=0.1 \mathrm{Mm}$, but allow $A_{\mathrm{v}}$ to vary in different simulations.

At the initial stage of the wave evolution, the initial pulse divides into counter-propagating waves. As a result of the rapid decrease in the equilibrium mass density, the upward propagating pulse grows in amplitude and quickly steepens into a shock. Figure 1 illustrates the spatial profile of $V(y)$ at $t=250 \mathrm{~s}$ for $A_{\mathrm{v}}=1 \mathrm{~km} \mathrm{~s}^{-1}$. At this time, the pulse reaches the level $y=23.5 \mathrm{Mm}$, while the secondary shock begins to form from the nonlinear wake at $y=2 \mathrm{Mm}$. Later on, this secondary shock propagates upwards, and is then followed by the next shock. This process repeats itself in time until the perturbation energy finally subsides to zero.

Figure 2 displays the temporal dynamics of the velocity that is collected at $y=20 \mathrm{Mm}$ for the initial pulse amplitude of $A_{\mathrm{v}}=1 \mathrm{~km} \mathrm{~s}^{-1}$ (top panel) and $A_{\mathrm{v}}=2 \mathrm{~km} \mathrm{~s}^{-1}$ (bottom panel). In the top panel, the arrival of the leading shock to the detection point occurs at $t \simeq 250 \mathrm{~s}$ and the second pulse reaches the detection point at $t \simeq 570 \mathrm{~s}$, i.e., after $\sim 320 \mathrm{~s}$. Thus, the nonlinear wake in the chromosphere excites quasi-periodic upwardly propagating pulses, which enter the corona and may cause the quasi-periodic intensity oscillations that have been observed in 


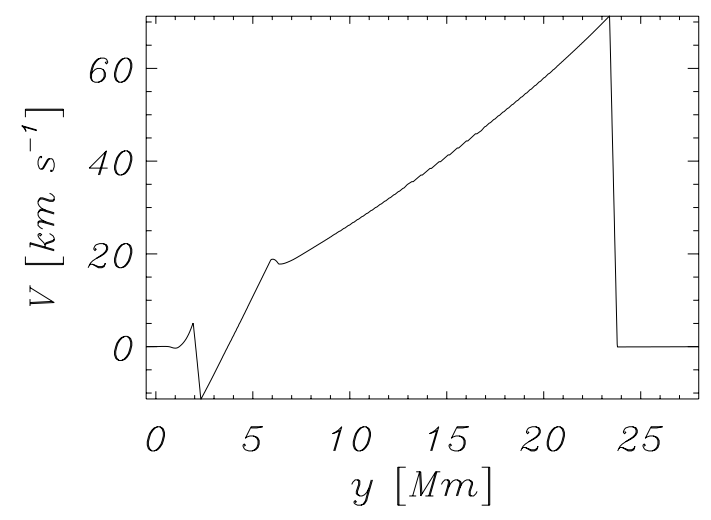

Fig. 1. Velocity (in units of $1 \mathrm{~km} \mathrm{~s}^{-1}$ ) profile versus height $y$ (in units of $1 \mathrm{Mm})$ at $t=250 \mathrm{~s}$ for $A_{\mathrm{v}}=1 \mathrm{~km} \mathrm{~s}^{-1}$. Here $y=0 \mathrm{Mm}(y \simeq 2.7 \mathrm{Mm})$ corresponds to the base of the photosphere (the transition region).
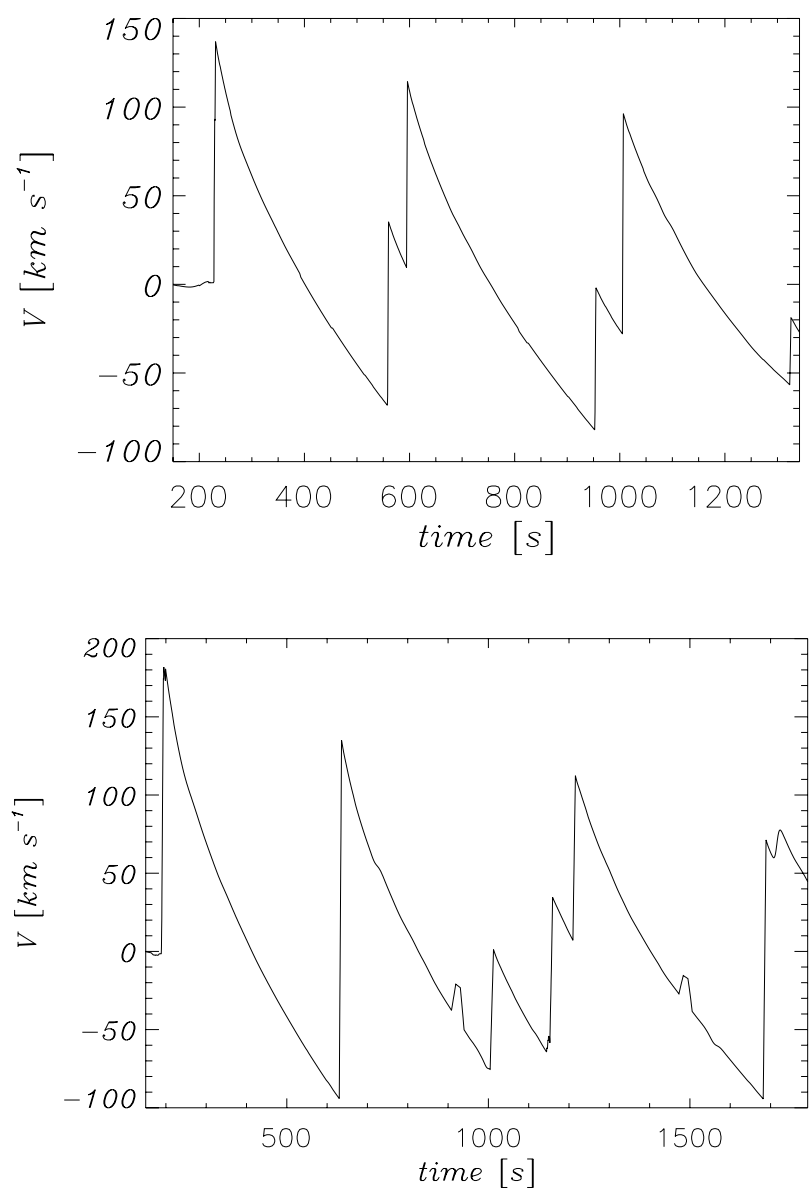

Fig. 2. Time signatures of $V$ (in units of $\mathrm{km} \mathrm{s}^{-1}$ ) collected at $y=20 \mathrm{Mm}$ for the case of $A_{\mathrm{v}}=1 \mathrm{~km} \mathrm{~s}^{-1}$ (top panel) and $A_{\mathrm{v}}=2 \mathrm{~km} \mathrm{~s}^{-1}$ (bottom panel). Time is expressed in units of $1 \mathrm{~s}$.

the imaging data. For $A_{\mathrm{v}}=1 \mathrm{~km} \mathrm{~s}^{-1}$, which is close to the typical granular velocity, the interval between the arrivals of neighboring shocks is nearly 5-min (Fig. 2, top). This means that the nonlinear wake produced in the realistic atmosphere and for the initial perturbations, corresponding to the solar granular velocity, exhibits about a 5-min wave period, i.e., it is longer than in the linear isothermal case $(\sim 3$-min in the chromosphere). Time signature for $A_{\mathrm{v}}=2 \mathrm{~km} \mathrm{~s}^{-1}$ shows that the leading shock arrives at the detection point at $t \simeq 190 \mathrm{~s}$, while the second shock reaches this point at $t \simeq 630 \mathrm{~s}$, i.e. after $\sim 440 \mathrm{~s}$ (Fig. 2, bottom panel). It is clearly seen that the interval between the arrival times of two consecutive shocks is longer for an initial pulse of larger amplitude.

\section{Discussion and summary}

Frequently observed $\sim 5$-min oscillations in the solar corona are often explained by the leakage of photospheric $\mathrm{p}$-modes along an inclined magnetic field. Vertically propagating acoustic waves with 5-min periods are evanescent because of the stratification of the solar atmosphere, since the chromospheric acoustic cutoff period is $\sim 3 \mathrm{~min}$ (Roberts 2004). However, acoustic-gravity waves have a lower cut-off frequency when they propagate at the angle about the vertical. This may allow the photospheric 5-min oscillations to be channeled along a stratified chromosphere and to penetrate into the corona (De Pontieu et al. 2005; Erdélyi et al. 2007; Fedun et al. 2009). To increase the cut-off period from 3 -min up to 5-min, the propagation angle (or magnetic field inclination) should be $\theta \sim 50^{\circ}(\cos \theta \approx 3 / 5)$. Therefore, the leakage of p-modes may only take place in particular regions of the solar atmosphere, where the magnetic field is significantly inclined in the chromosphere.

In this paper, we have proposed an alternative mechanism to explain the observed oscillations in the solar corona, which is based on the rebound shock model of Hollweg (1982). We have numerically solved the full set of Euler equations for both the realistic VAL-C temperature profile and a Gaussian velocity pulse launched within the photosphere. We found that velocity pulses, originating from granules or magnetic reconnection in the lower regions, lead to different responses of the chromosphere/transition region than the periodic acoustic waves resulting from p-modes. We note that the energy of granular motions is higher than the energy of p-modes, hence the impulsively triggered waves should have more power than those triggered by a periodic driver.

The numerical simulations show that as a result of the rapid decrease in the equilibrium mass density the initial velocity pulse quickly steepens into a shock. The shock propagates into the corona, while the nonlinear wake is formed in the chromosphere by the atmospheric stratification. This nonlinear wake leads to consecutive shocks, as first shown by Hollweg (1982). The interval between the arrival times of two consecutive shocks depends on the amplitude of the initial pulse; a stronger pulse leads to longer intervals. The initial pulse with the granular velocity of $1 \mathrm{~km} \mathrm{~s}^{-1}$ leads to $\sim 5$-min intervals between consecutive shocks. Therefore, the quasi-periodic arrival of consecutive shocks in the solar corona may generate the intensity oscillations with a period close to the interval between the shocks.

We have implemented a simple 1D analytical model to avoid the propagation of acoustic oscillations at any non-zero angle to the vertical. We have shown that purely vertically propagating pulses may lead to quasi 5-min oscillations in the corona caused by consecutive shocks.

The one-dimensional propagation of acoustic waves is justified for purely vertical magnetic field. In this case, acoustic waves represent slow magneto-acoustic waves for low plasma $\beta \sim c_{\mathrm{S}}^{2} / v_{\mathrm{A}}^{2}<1$ and fast magneto-acoustic waves for high plasma $\beta>1$, where $v_{\mathrm{A}}$ is the Alfvén speed. In the solar photosphere, $\beta$ is larger than unity, but rapidly decreases as the mass density falls off with height (as consequently the Alfvén speed 
increases). It becomes smaller than unity in the chromosphere (Gary 2001) tending to unity somewhere between the photosphere and the chromosphere, this surface needing to be thinner than the width of the chromosphere. The linear fast and slow magneto-acoustic waves are coupled near the level of $\beta \sim 1$ when they propagate obliquely to the magnetic field (Rosenthal et al. 2002; Bogdan et al. 2003). However, these waves remain purely acoustic for parallel propagation unless tube dispersive effects are taken into account. Hence, a pulse propagating along the vertical magnetic field may not feel the $\beta \sim 1$ surface. On the other hand, the acoustic wake, which is formed behind the pulse and oscillates along the magnetic field, may lead to a nonlinear transfer of energy into Alfvén waves near the $\beta \sim 1$ region (Zaqarashvili \& Roberts 2006; Kuridze \& Zaqarashvili 2008). This effect may be discernible if one included magnetic fields in numerical simulations. Some of the oscillation energy may then be transformed into transverse oscillations near this region, although most of energy will remain in longitudinal oscillations because of the thinness of the $\beta \sim 1$ region. Therefore, the $\beta \sim 1$ region may not significantly affect the formation of rebound shocks in the chromosphere and consequently quasiperiodic acoustic oscillations in the lower corona. However, a two-dimensional analysis and the inclusion of magnetic fields are necessary to completely understand the proposed scenario. The first step in this direction was achieved by Murawski \& Zaqarashvili (2010) by modeling the spicule formation, although they considered a simple temperature profile covering only the chromosphere-corona. The plasma $\beta$ was considered less than unity along the whole simulation region, hence the effects of the $\beta \sim 1$ region were absent in these simulations. We intend to consider the rebound shock model in the case of a magnetic field and a realistic temperature profile in the future.

An important consequence of the rebound shock model is that the interval between consecutive shocks depends on the initial amplitude of pulse (see Fig. 2). The smaller amplitude pulses lead to shorter intervals between consecutive shocks with a lower limit of 3-min, which is the linear acoustic cut-off period of the solar atmosphere. The interval is longer for stronger initial pulses being $\sim 5$-min for $1 \mathrm{~km} \mathrm{~s}^{-1}$ in the photosphere. The rebound shock model then can predict the longer period oscillations above the regions of strong granular power. The granulation is suppressed in strong magnetic field regions of the photosphere (e.g. sunspots), where the initial pulses should thus have smaller amplitudes, leading to an oscillation close to the near cut-off frequency. The strong magnetic field regions (sunspots, magnetic network cores) indeed predominantly undergo 3-min oscillations in the chromosphere. We note that some observations also provide evidence of 3-min oscillations in the solar corona above sunspots (De Moortel et al. 2002) and other magnetic structures (Lin et al. 2005). These oscillations can be excited as a consequence of consecutive shocks caused by chromospheric 3-min oscillations.

As a result, we expect $\sim 5$-min oscillations to be detected above the regions where the granular energy is significant, i.e., the quiet Sun and the surroundings of active regions/magnetic network. This is consistent with observations. On the other hand, the detection of 5-min oscillations in the quiet corona is not an easy task because of the relatively weak intensity of coronal lines. However, a careful analysis can still be performed. One should keep in mind that the dynamics of acoustic waves in the field-free regions and along the vertical magnetic field could be quite different from that discussed above. An initial acoustic pulse may spread out horizontally in field-free regions, while almost all of energy would be guided along the field lines in magnetic structures. Therefore, the amplitude of intensity oscillations might be smaller in the quiet corona than near active regions and chromospheric network cores. However, the divergence of the magnetic field and greater thermal conduction may significantly weaken the amplitude of coronal oscillations, which seem to be quite strong and non-linear in Figs. 1 and 2. The strong slow wave pulses may then become almost linear as they penetrate the corona, as seen by observations. In our numerical model, we therefore need to include both a magnetic field and thermal conduction (at least, in the coronal part of the atmosphere), which will be done in future studies.

We note that the granular velocities may have values in the range $0.5-2 \mathrm{~km} \mathrm{~s}^{-1}$ with a peak at $1 \mathrm{~km} \mathrm{~s}^{-1}$. As the interval between consecutive shocks strongly depends on the amplitude of the initial pulse, the resulted coronal oscillations may then take values in the range 4-7 min with a peak at 5-min. Indeed, the wavelet analysis of coronal line images obtained by Hinode/EIS indicates that the oscillation power of coronal oscillations is concentrated at a period in a range of 4-6 min (Wang et al. 2009), which is fully consistent with our theory.

Our simulations were performed for an isolated pulse in order to show clearly the effect of rebound shocks. However, the solar photosphere is very dynamic, hence the initial pulse is probably followed by other pulses. The subsequent pulse coming from the photosphere may also trigger consecutive shocks in the chromosphere. The interaction between rebound shocks formed by different photospheric pulses may set up complex dynamics in the chromospheric plasma that has an immediate influence on the lower corona. Therefore, the coronal oscillations may have a broad spectrum as we discussed in the previous paragraph. On the other hand, if the mean interval between subsequent initial pulses were close to the mean interval between consecutive shocks, then a resonance might occur in the chromosphere. This investigation will be the subject of a future study.

The excitation of coronal oscillations due to the leakage of p-modes may only occur along significantly inclined magnetic field in the chromosphere (to reduce the cut-off frequency). The magnetic field lines, which are significantly inclined from the vertical in the chromosphere, may not reach the corona at all. Therefore, the p-mode leakage may not occur in cases represented by the real geometry of the active region magnetic field. In contrast, the rebound shock mechanism may operate in a magnetic field of any geometry including the purely vertical field lines. Therefore, the excitation of 5-min oscillations in the solar corona by photospheric impulsive drivers has a larger area of application than that of by p-modes. Future more sophisticated models may shed light on the excitation of coronal acoustic waves.

Our conclusions are:

(a) A velocity pulse that is initially launched at the photospheric level (due to granules or reconnection) quickly steepens into a shock and can penetrate into the corona, while a nonlinear wake that is formed behind this shock leads to consecutive shocks in the chromosphere.

(b) For the initial photospheric pulse amplitude of $1 \mathrm{~km} \mathrm{~s}^{-1}$, the time interval between two consecutive shocks is $\sim 5$-min; the consecutive shocks propagate upwards and may cause the observed 5-min intensity oscillations in the solar corona.

(c) Overall, the observed $\sim 5$-min oscillations in the solar corona could be caused by impulsive photospheric perturbations (i.e., convection, reconnection) not necessarily by p-modes. 
T. V. Zaqarashvili et al.: 5-min oscillations in the solar corona

Acknowledgements. The authors express their thanks to the unknown referee for his/her constructive comments. The work of T.Z. and M.K. was supported by the Austrian Fond zur Förderung der Wissenschaftlichen Forschung (project P21197-N16). The work of K.M. was supported by the Polish Ministry of Science (the grant for years 2007-2010). T.Z. was also supported by the Georgian National Science Foundation grant GNSF/ST09/4-310. The software used in this work was in part developed by the DOE-supported ASC/Alliance Center for Astrophysical Thermonuclear Flashes at the University of Chicago.

\section{References}

Bel, N., \& Leroy, B. 1977, A\&A, 55, 239

Bogdan, T. J., Hansteen, M., Carlsson, V., et al. 2003, ApJ, 599, 626

Carlsson, M., \& Stein, R. F. 1997, ApJ, 481, 500

De Moortel, I., Ireland, J., \& Walsh, R. W. 2000, A\&A, 355, L23

De Moortel, I., Ireland, J., Hood, A. W., \& Walsh, R. W. 2002, A\&A, 387, L13

De Pontieu, B., Erdélyi, R., \& De Moortel, I. 2005, ApJ, 624, L61

Dubey, A., Antypas, K., Ganapathy, M. K., \& Reid, L. B. 2009, Extensible

component-based architecture for FLASH, a massively parallel, multiphysics

simulation code, ed. K. Riley, D. Sheeler, A. Siegel, \& K. Weide, Parallel Computing, 35

Erdélyi, R., Malins, C., Tóth, G., \& de Pontieu, B. 2007, A\&A, 467, 1299

Fedun, V., Erdélyi, R., \& Shelyag, S. 2009, Sol. Phys., 258, 219

Gary, G. A. 2001, Sol. Phys., 203, 71
Hollweg, J. V. 1982, ApJ, 257, 345

Kuridze, D., \& Zaqarashvili, T. V. 2008, J. Atmos. Solar-Terres. Phys., 70, 351

Kuridze, D., Zaqarashvili, T. V., Shergelashvili, B. M., \& Poedts, S. 2009, A\&A, 505,763

Lamb, H. 1908, Proc. London Math. Soc, 7, 122

Lin, C. H., Banerjee, D., Doyle, J. G., \& O'Shea, E. 2005, A\&A, 444, 585

Lin, C. H., Banerjee, D., O’Shea, E., \& Doyle, J. G. 2006, A\&A, 460, 597

Marsh, M. S., Walsh, R. W., De Moortel, I., \& Ireland, J. 2003, A\&A, 404, L37

McIntosh, S. W., \& Jefferies, S. W. 2006, ApJ, 647, L77

Murawski, K., \& Zaqarashvili, T. 2010, A\&A, 519, A8

Musielak, Z. E., Musielak, D. E., \& Mobashi, H. 2006, Phys. Rev. E, 036612

Narain, U., \& Ulmschneider, P. 1990, Space Sci. Rev., 54, 377

Narain, U., \& Ulmschneider, P. 1996, Space Sci. Rev., 75, 453

Rae, I. C., \& Roberts, B. 2002, ApJ, 256, 761

Roberts, B. 2004, In Proc. SOHO 13 Waves, Oscillations and Small-Scale Transient Events in the Solar Atmosphere: A Joint View from SOHO and TRACE, Palma de Mallorca, Spain, ESA SP-547, 1

Rosenthal, C. S., Bogdan, T. J., Carlsson, M., et al. 2002, ApJ, 564, 508

Ruderman, M. S. 2006, Phyl. Trans, R. Soc. A, 364, 485

Srivastava, A. K., Kuridze, D., Zaqarashvili, T. V., \& Dwivedi, B. N. 2008, A\&A, 481, L95

Vernazza, J. E., Avrett, E. H., \& Loeser, R. 1981, ApJ, 45, 635

Wang, T. J., Ofman, L., \& Davila, J. M. 2009, ApJ, 696, 1448

Zaqarashvili, T. V., \& Roberts, B. 2006, A\&A 452, 1053 